\title{
A system-theoretical evaluation method for the rever- beration time of an acoustically coupled room system
}

\author{
Mitsuo Ohta, ${ }^{*}$ Hiroshi Yamada, ${ }^{* *}$ and Hirofumi Iwashige*** \\ *Faculty of Engineering, Kinki University, \\ Umenobe 1, Takaya, Higashi-Hiroshima, 729-17 Japan \\ **Faculty of Engineering, University of East Asia, \\ Ichinomiya-Gakuencho 2-1, Shimonoseki, 751 Japan \\ ***Faculty of Education, Hiroshima University, \\ Kagamiyama 1, Higashi-Hiroshima, 724 Japan
}

(Received 25 February 1994)

\begin{abstract}
A reverberation time is very important for evaluating the reverberation characteristics of a room and the sound power of an acoustic source. The definition of reverberation time is given originally for an acoustically isolated single room. On the other hand, in an acoustically coupled room, respective reverberation times of individual rooms are fairly different from independently measured reverberation times without mutual power flow interactions through apertures or acoustic insulation walls. In this paper, first, a general type characteristic expression of acoustically coupled rooms is established as a power flow system consisting of several subsystems, in close connection with a Statistical Energy Analysis method, corresponding to a kind of system equation in a state estimation theory. On the basis of a solution of this system equation, new type six different methods are proposed on trial by introducing various type evaluation criteria so as to evaluate the reverberation time of the acoustically coupled room system, especially from various type system-theoretical viewpoints. Finally, the effectiveness of the proposed theory is experimentally confirmed by applying it to the actual coupled acoustic rooms and the above six evaluation methods are compared with each other.
\end{abstract}

Keywords: Reverberation time, Coupled room, Matrix norm, Matrix measure, Systemtheoretical viewpoint

PACS number: $43.55 . \mathrm{Br}$

\section{INTRODUCTION}

Not to say, a reverberation time is very important for evaluating the reverberation characteristics of a room and the sound power of an acoustic source. In general, as is well-known, a reverberation time of room is defined as the time interval in which the reverberation level drops down by $60 \mathrm{~dB}$ and experimentally measured from the actual decay curve. As is well-known, this definition is given, however, originally for an acoustically isolated single room.

On the other hand, an acoustically coupled room $^{1,2)}$ is functionally given as a system composed of virtually separated rooms, connected by relatively small apertures or by acoustically transparent walls. Owing to power flow interactions between arbitrary two rooms through the apertures or acoustic walls, a respective reverberation time of individual room in such a coupled room system is fairly different from independently measured reverberation times without mutual power flow interactions. But, until now, a considerable much researches for evaluating the reverberation characteristics of a coupled room were reported only in an extension form of the standard evaluation method for a single room..$^{3-8)}$

In this paper, first, a general type characteristic 
expression of acoustically coupled rooms is established as a power flow system consisting of several subsystems, in close connection with a well-known Statistical Energy Analysis method, corresponding to a kind of system equation in a state estimation theory. On the basis of a solution of this system equation, new type six different methods are proposed on trial by introducing various type evaluation criteria so as to evaluate the reverberation time of the acoustically coupled room system, especially from various type system-theoretical viewpoints. Thus, not only an extended method of conventional standard evaluation but also various type synthetic evaluation methods can be found. Finally, the effectiveness of the proposed theory is experimentally confirmed by applying it to the actual coupled acoustic rooms and the above six evaluation methods are compared with each other under various type different conditions of acoustical coupling.

\section{THEORETICAL CONSIDERATIONS}

\subsection{Matrix Equation of Power Flow for Acoustical-} ly Coupled Rooms

Let us consider an acoustically coupled room consisting of $n$ individual rooms in the form of total power flow system with $n$ subsystems as shown in Fig. 1. It is assumed that each room $\mathbf{R}_{i}$ has a sound source with an output power $W_{i}$ and an external noise is transmitted into only room $R_{1}$ through the window or the aperture for simplicity.

According to Statistical Energy Analysis (abr. SEA) method, ${ }^{9)}$ simultaneous equations of power balance among $n$ rooms are expressed as follows:

$$
\begin{aligned}
V_{1} \frac{d E_{1}}{d t}= & W_{0}-\pi_{1,0}-\pi_{1}-\pi_{1,2}+\pi_{2,1}+W_{1}, \\
V_{i} \frac{d E_{i}}{d t}= & \pi_{i-1, i}-\pi_{i, i-1}-\pi_{i}-\pi_{i, i+1} \\
& \quad+\pi_{i+1, i}+W_{i}, \quad(i=2,3, \cdots, n-1) \\
V_{n} \frac{d E_{n}}{d t}= & \pi_{n-1, n}-\pi_{n, n-1}-\pi_{n}+W_{n},
\end{aligned}
$$

where

$V_{i}$ : air volume of room $\mathrm{R}_{i}\left[\mathrm{~m}^{3}\right]$,

$E_{i}\left(\equiv E_{i}(t)\right)$ : sound energy density in room $\mathrm{R}_{i}$ $\left[\mathrm{J} / \mathrm{m}^{3}\right]$,

$\pi_{i}\left(\equiv \pi_{i}(t)\right)$ : sound energy lost through walls in room $\mathrm{R}_{i}$ per second $[\mathrm{J} / \mathrm{s}]$,

$\pi_{i, j}\left(\equiv \pi_{i, j}(t)\right)$ : sound energy transmitted from room $\mathbf{R}_{i}$ to room $\mathbf{R}_{j}$ per second $[\mathrm{J} / \mathrm{s}]$.

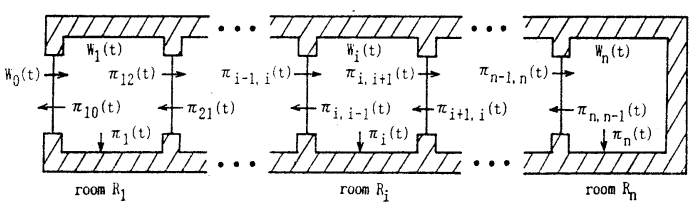

Fig. 1 Acoustically coupled series rooms.

For establishing a basic theory to evaluate the acoustic characteristics of this coupled room system, let us assume that the sound field in each room is a diffuse sound field and that the sound energy of each room is transmitted into the adjoining rooms only through its apertures or connecting walls. At this time, considering that sound energy incidents to a unit area of space in a unit time is given by $c E_{i}(t) / 4$ in a perfect diffuse field, where $c$ is the sound velocity, Eqs. (1a)-(1c) can be organized into a matrix equation as follows:

$$
\frac{d E(t)}{d t}=A E(t)+B u(t),
$$

where

$$
\begin{aligned}
& \boldsymbol{E}(t)=\left[E_{1}(t), E_{2}(t), \cdots, E_{n}(t)\right]^{\mathrm{T}}, \\
& \boldsymbol{u}(t)=\left[W_{0}(t)+W_{1}(t), W_{2}(t), \cdots, W_{n}(t)\right]^{\mathrm{T}},
\end{aligned}
$$

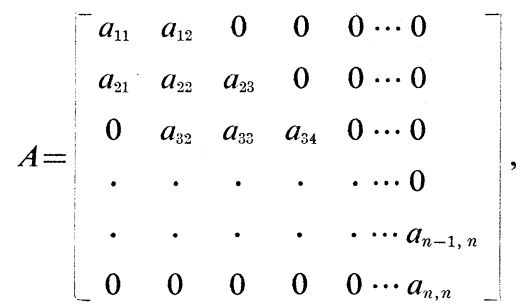

$$
\begin{aligned}
& {\left[\frac{1}{V_{1}} \quad 0 \quad 0 \quad \cdots \quad 0\right.} \\
& B=\left[\begin{array}{ccccc}
0 & \frac{1}{V_{2}} & 0 & \cdots & 0 \\
. & . & . & \cdots & 0 \\
0 & 0 & 0 & \cdots & \frac{1}{V_{n}}
\end{array}\right], \\
& a_{i j}=\frac{c S_{j i} \tau_{j i}}{4 V_{i}} \quad(i \neq j, i, j=1,2, \cdots, n), \\
& a_{i i}=-\frac{c}{4 V_{i}}\left\{S_{i} \alpha_{i}+S_{i, i-1}\left(\alpha_{i, i-1}+\tau_{i, i-1}\right)\right. \\
& \left.+S_{i, i+1}\left(\alpha_{i, i+1}+\tau_{i, i+1}\right)\right\} \quad(i=1,2, \cdots, n-1), \\
& a_{n n}=-\frac{c}{4 V_{n}}\left\{S_{n} \alpha_{n}+S_{n, n-1}\left(\alpha_{n, n-1}+\tau_{n, n-1}\right)\right\}
\end{aligned}
$$




\section{OHTA et al.: SYSTEM-THEORETICAL EVALUATION METHOD OF COUPLED ROOM}

with

$S_{i j}$ : area of partition between $\mathbf{R}_{i}$ and $\mathrm{R}_{j}\left(S_{i j}=\right.$ $\left.S_{j i}\right)$,

$\alpha_{i j}$ : sound absorption coefficient of the surface of partition between $\mathrm{R}_{i}$ and $\mathrm{R}_{j}$,

$S_{i}$ : inner surface area of room $\mathrm{R}_{i}$ excepting $S_{i, i-1}$ and $S_{i, i+1}$,

$\alpha_{i}$ : mean sound absorption coefficient of the inner surface $S_{i}$,

$\tau_{i j}$ : transmission coefficient of the surface of partition between $\mathrm{R}_{i}$ and $\mathrm{R}_{j}\left(\tau_{i j}=\tau_{j i}\right)$.

Hereupon, the following points must be noticed:

(1) The $n$-dimensional state vector $\boldsymbol{E}(t)$ denotes an $n$ set of sound energy density in each room.

(2) The $n$-dimensional vector $\boldsymbol{u}(t)$ denotes an $n$ set of output power of sound source in each room.

(3) The coefficient matrix $A$ is a tridiagonal matrix and the matrix $B$ is a diagonal matrix.

In the actual living situation, it is difficult to measure the accurate values of each sound absorption coefficient $\alpha_{i}$ and the transmission coefficient $\tau_{i j}$. Accordingly, it is difficult to calculate the accurate values of element $a_{i j}$ in matrix $A$ from Eq. (4) on the basis of the internal mechanism of a coupled room. Therefore, we have proposed a new trial of parameter identification for $a_{i j}$ in the above matrix state equation.

\subsection{Solution of Matrix Type Reverberation Equa-} tion

As mentioned in the previous section, such a coupled room can be considered as a power flow system. In this section, the solution of the matrix system equation, Eq. (1), is discussed especially from a system-theoretical viewpoint.

Following to the well-known solution of a timeinvariant linear type differential equation in the state space method, a solution of Eq. (1) is given directly as follows:

$$
\boldsymbol{E}(t)=\Phi(t) \boldsymbol{E}_{0}+\int_{0}^{t} \Phi(t-\tau) B \boldsymbol{u}(\tau) d \tau,
$$

where

$$
\begin{aligned}
& \boldsymbol{E}(t)=\left[E_{1}(t), E_{2}(t), \cdots, E_{n}(t)\right]^{\mathrm{T}}, \\
& \boldsymbol{E}_{0}=\boldsymbol{E}(0)=\left[E_{1}(0), E_{2}(0), \cdots, E_{n}(0)\right]^{\mathrm{T}}
\end{aligned}
$$

and

$$
\Phi(t)=e^{A t} .
$$

Because $a_{i-1, i}$ and $a_{i, i-1}$ are positive from Eq. (4), matrix $A$ has $n$ different real eigenvalues of negative value. Let $n$ real eigenvalues be $-\lambda_{1},-\lambda_{2}, \cdots$, $-\lambda_{n}$, where these are classified as $0<\lambda_{1} \leq \lambda_{2} \leq \cdots \leq$ $\lambda_{n}$. After employing a well-known Sylvester's expansion theorem, the transition matrix $\Phi(t)$ can be easily expressed as follows:

$$
\Phi(t)=\sum_{i=1}^{n} e^{-\lambda_{i} t} \prod_{j=1, j \neq i}^{n} \frac{A+\lambda_{i} I}{-\lambda_{i}+\lambda_{j}} .
$$

Next, the stationary response $\boldsymbol{E}_{0}$ of this system for a stationary input $\boldsymbol{u}_{0} \equiv \boldsymbol{u}(0)$ is given by setting $d \boldsymbol{E}(t) /$ $d t=\mathbf{0}$ in Eq. (2) as follows:

$$
\boldsymbol{E}_{0}=-A^{-1} B \boldsymbol{u}_{0} .
$$

Let all of the sources stop at the same time when the sound field in each coupled room reaches at a stationary state. After that, $\boldsymbol{E}(t)$ decays exponentially as follows:

$$
\boldsymbol{E}(t)=\Phi(t) \boldsymbol{E}_{0}=-e^{A t} A^{-1} B \boldsymbol{u}_{0} .
$$

It is easily shown from Eq. (6) that every element $E_{i}(t)$ of vector $\boldsymbol{E}(t)$ consists of $n$ different damping components. Hereupon, we must notice that a matrix $e^{A t} A^{-1} B$ shows a symmetry.

\subsection{New Evaluation Methods for Reverberation} Time of Coupled Room System

In this section, six different type methods are newly proposed on trial for evaluating the reverberation time of a coupled room system especially from various type system-theoretical viewpoints. ${ }^{11-14)}$

(Method 1) A synthetic evaluation method

By regarding many coupled rooms as a total system consisting of several subsystems, the following synthetic evaluation method of reverberation time can be proposed.

When all of sound sources into the coupled rooms stop at $t=0$, the vector $\boldsymbol{E}(t)$ of reverberation process, is given as mentioned in the previous section:

$$
\boldsymbol{E}(t)=\Phi(t) \boldsymbol{E}_{0} .
$$

A time $T$ satisfying the following equation can be synthetically employed as a reverberation time of a coupled room system with the sound power flow interactions:

$$
10 \log _{10} \frac{\|\boldsymbol{E}(T)\|}{\left\|\boldsymbol{E}_{0}\right\|}=-60,
$$

where $\|\cdot\|$ denotes a vector norm. It is noteworthy that $T$ satisfying Eq. (10) especially for one dimensional state variable $E(T)$ agrees with the conventional definition of a reverberation time due to Sabine. 
There are various ways on how to choose the vector norm according to the engineering needs. For example, every room should be treated with an equal weight, or each room should be reasonably treated with a particular weight. At this time, of course, $T$ 's obtained in the different ways of selecting vector norm are different from one another and from a reverberation time obtained by the traditional way. Once after a vector norm is selected in advance, the following relationship can be set by newly introducing a matrix norm and a measure of matrix determined by the vector norm:

$$
\begin{gathered}
\max \{-\mu(-\Phi(T)),-\mu(\Phi(T))\} \\
\leq \frac{\|\boldsymbol{E}(T)\|}{\left\|\boldsymbol{E}_{0}\right\|} \leq\|\Phi(T)\|,
\end{gathered}
$$

where $\mu(A)$ is a measure of matrix $A$ and $\|A\|$ is a matrix norm of matrix $A$.

In other words, it can be expected that Eq. (11) gives a certain measure of allowance for the estimation of $T$. In particular, when an Euclidean norm:

$$
\|\boldsymbol{x}\|_{2}=\sqrt{\sum_{i=1}^{n}\left|x_{i}\right|^{2}}
$$

is selected for the vector norm in Eq. (10) and matrix $A$ is a real symmetric matrix, the corresponding measure of matrix and matrix norm for this matrix $A$ can be given as follows:

$$
\begin{aligned}
& \mu_{2}(A)=\max _{i}\left\{\lambda_{i}\left(\frac{A^{*}+A}{2}\right)\right\}, \\
& \|A\|_{2}=\sqrt{\max _{i}\left\{\lambda_{i}\left(A^{*} A\right)\right\},}
\end{aligned}
$$

where $\lambda_{i}(A)$ denotes the $i$-th eigenvalue of matrix $A$ and $A^{*}$ denotes a conjugate transpose matrix of $A$.

Finally, Eq. (11) can be rewritten in a more concrete forms as follows:

$$
e^{-i_{n} T} \leq \frac{\|\boldsymbol{E}(T)\|_{2}}{\left\|\boldsymbol{E}_{0}\right\|_{2}} \leq e^{-\lambda_{1} T},
$$

where it is assumed that those eigenvalues are classified as $\lambda_{1} \leq \lambda_{2} \leq \cdots \leq \lambda_{n}$.

(Method 2) A conventional evaluation method

In this method, though there are acoustically power flow interactions among the rooms, the reverberation time of each room is separately and conventionally measured as a time to decay $60 \mathrm{~dB}$ from an initial level based on a measured reverberation curve.

(Method 3) An evaluation method with a safety factor
Equations (5) and (6) show that $n$ kinds of reverberation processes in $n$ rooms have respectively the proper pattern of exponentially decay curve.

In this method, as one of six evaluation methods for a whole coupled room system, a reverberation time of a coupled room is evaluated with a safety factor by use of the most slowly decaying curve among $n$ exponentially decaying curves as follows:

$$
T=\frac{6 \ln 10}{\min _{i} \lambda_{i}} .
$$

Note that this method gives a representative reverberation time for a coupled room system.

(Method 4) An approximated evaluation method of locally exponential type

Based on the above consideration, a reverberation process in the $i$-th room $\mathbf{R}_{i}$ can be obviously approximated by an exponentially damping function, $E_{i 0} e^{-\mu i t}$. Let the approximated reverberation process be $\hat{E}(t)$. Then, $\hat{E}(t)$ can be expressed as follows:

$$
\hat{\boldsymbol{E}}(t)=\operatorname{diag}\left[e^{-\prime_{1} t}, e^{-\mu_{2} t}, \cdots, e^{-\mu_{n} t}\right] \boldsymbol{E}_{0} .
$$

Hereupon, the constant $\mu_{i}$ is determined so as to make the inclination of $e^{-\mu i t}$ equal approximately to the inclination of $E_{i}(t)$ at $t=0$. That is, a reberberation time of the room $\mathbf{R}_{i}$ is approximately given as follows:

$$
T_{i}=\frac{6 \ln 10}{\mu_{i}} .
$$

(Method 5) An approximated evaluation method of integrated exponential type

As another evaluation method, a constant $\mu_{i}$ can be determined so as to make the integrated value of $E_{i 0} e^{-\mu_{i} t}$ equal to the integrated value of $E_{i}(t)$ in a whole time domain $[0, \infty)$, as follows:

$$
\int_{0}^{\infty} \boldsymbol{E}(t) d t=\int_{0}^{\infty} \operatorname{diag}\left[e^{-\mu_{1} t}, e^{-\mu_{2} t}, \cdots, e^{-\mu_{n} t}\right] \boldsymbol{E}_{0} d t .
$$

That is, a reverberation time of the room $\mathrm{R}_{i}$ can be given as follows:

$$
\begin{aligned}
& {\left[T_{1}, T_{2}, \cdots, T_{n}\right]^{\mathrm{T}}} \\
& \quad=-6 \ln 10\left\{\operatorname{diag}\left[E_{10}, E_{20}, \cdots, E_{n 0}\right]\right\}^{-1} A^{-1} E_{0} .
\end{aligned}
$$

(Method 6) Ziegler-Nichols' type approximated evaluation method

On the other hand, a reverberation time $T_{i}$ in the room $\mathrm{R}_{i}$ can be determined based on the inclination at the inflection point on the decay curve drawn by Eq. (6). This evaluation method is the same as the well-known Ziegler-Nichols' approximation in the field of automatic control system (see Fig. 2). 


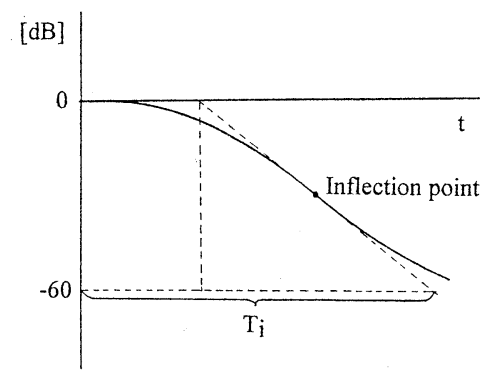

Fig. 2 Ziegler-Nichols' approximation method.

\section{EXPERIMENTAL CONSIDERATIONS}

\subsection{Experimental Method}

In the experimental study, the measurement of a reverberation time has been carried out especially in a coupled room system with two kind of individual reverberant rooms as shown in Fig. 3. A room $R_{1}$ has an air volume of $50.2 \mathrm{~m}^{3}$ and a room $\mathrm{R}_{2}$ has an air volume of $24.6 \mathrm{~m}^{3}$. The aperture of the wall between $R_{1}$ and $R_{2}$ has an area of $1.74 \mathrm{~m} \times 0.84 \mathrm{~m}$.

The following three cases for this aperture have been considered for measuring quantitatively the acoustic influences of strength of mutual coupling:

(Case 1) fully opened,

(Case 2) 1/8 opened,

(Case 3) closed with a lauan board of $3 \mathrm{~mm}$ thickness.

The sound source signal has been an octave band white noise with a center frequency $500 \mathrm{~Hz}$. Each room can be regarded respectively as a complete diffuse sound field (See Appendix for the experimental verification of complete diffuseness of each room). The sampling interval of the measurement has been $1 / 6 \mathrm{~s}$ and 500 data have been used and then averaged to obtain individually the stationary energy densities in two rooms. Moreover, the experimental data have been observed for several locations of the sound source and the microphones in each room, and have been spatially averaged for finding successfully a complete diffuseness in our experiment.

\subsection{Results of Parameter Estimation}

The method of measuring an output power of source can be first briefly explained as follows.

Let us assume that the sound source to be measured emits a power $W$ in a reverberant room. At this time, an energy density in the room can be ex-

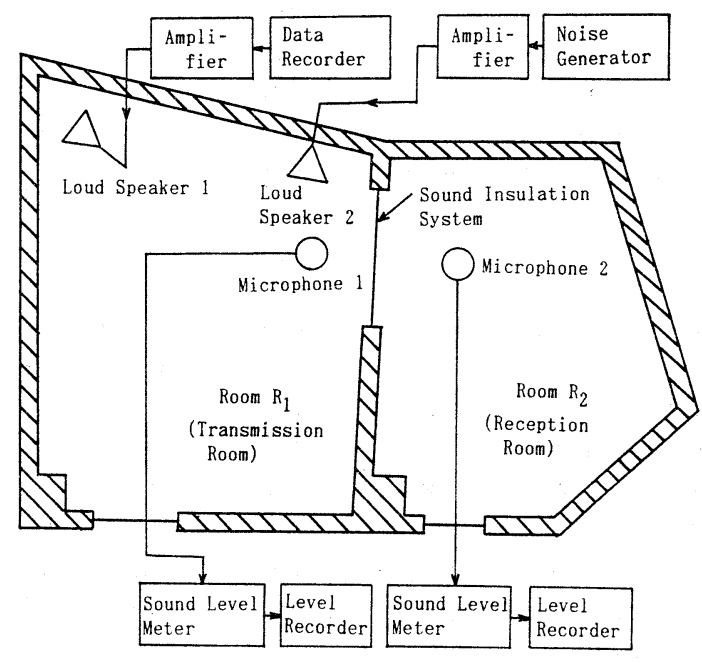

Fig. 3 Block diagram experiment.

pressed as follows:

$$
\begin{gathered}
V \frac{d E(t)}{d t}=W-K E(t), \\
K=\frac{c \alpha S}{4},
\end{gathered}
$$

where

$V:$ air volume of the room,

$c$ : sound velocity,

$\alpha$ : mean sound absorption coefficient,

$S$ : inner surface area of the room.

After stopping the sound source emission when the energy density in the room reaches at a stationary state, $E_{0}=W / K$, the following solution of the above reverberation equation can be derived:

$$
E(t)=E_{0} e^{-(K / V) t} .
$$

As is well-known, from this equation, a reverberation time of the room is given as follows:

$$
T=6 \ln 10 \cdot \frac{V}{K} \text {. }
$$

Finally, from Eqs. (20) and (22), the output power of sound source can be estimated as follows:

$$
W=6 \ln 10 \cdot \frac{V}{T} E_{0} .
$$

That is to say, the sound output power $W$ can be measured by measuring a reverberation time and the stationary energy density $E_{0}$. The estimation of $W$ has been carried out in a room $R_{1}$ in a typical case where absolutely isolated from a room $R_{2}$ and the 
estimated value of $W$ has been 0.466 watt.

From our another related research ${ }^{10)}$ for estimating the parameter matrix $A$ in the system Eq. (2), an estimated parameter matrix $A$ is given by a deterministic estimation method as follows:

$$
A=-\operatorname{diag}\left[\frac{u_{1}}{V_{1}}, \frac{u_{2}}{V_{2}}\right] \cdot\left[E_{1}, E_{2}\right]^{-1},
$$

where $\boldsymbol{E}_{i}$ denotes a vector of stationary energy densities of rooms $R_{1}$ and $R_{2}$ when a sound source with an input $u_{i}$ is placed in a room $\mathrm{R}_{i}$. Substituting the value of $W$ for $u_{1}$ or $u_{2}$ in Eq. (24) (cf. Eqs. (2) and (3) when $d \boldsymbol{E} / d t=\mathbf{0}$ (stationary)) and observing the actual data of $\boldsymbol{E}_{i}$, the parameter matrix $A$ has been concretely estimated. The values of $\boldsymbol{E}_{1}, \boldsymbol{E}_{2}, A$ and the eigenvalues $\left(\lambda_{1}, \lambda_{2}\right)$ of $A$ are shown in the previous three cases as follows:

(Case 1)

$$
\begin{aligned}
\boldsymbol{E}_{1} & =\left[\begin{array}{l}
2.04 \times 10^{-3} \\
1.26 \times 10^{-3}
\end{array}\right], \quad \boldsymbol{E}_{2}=\left[\begin{array}{l}
8.71 \times 10^{-3} \\
3.02 \times 10^{-3}
\end{array}\right], \\
A & =\left[\begin{array}{rr}
-5.52 & 1.59 \\
4.70 & -7.62
\end{array}\right], \quad\left[\begin{array}{l}
\lambda_{1} \\
\lambda_{2}
\end{array}\right]=\left[\begin{array}{l}
-3.64 \\
-9.50
\end{array}\right],
\end{aligned}
$$

(Case 2)

$$
\begin{aligned}
\boldsymbol{E}_{1} & =\left[\begin{array}{c}
2.34 \times 10^{-3} \\
3.16 \times 10^{-4}
\end{array}\right], \quad \boldsymbol{E}_{2}=\left[\begin{array}{l}
3.16 \times 10^{-4} \\
4.17 \times 10^{-3}
\end{array}\right], \\
A & =\left[\begin{array}{cc}
-4.00 & 0.303 \\
0.618 & -4.59
\end{array}\right], \quad\left[\begin{array}{l}
\lambda_{1} \\
\lambda_{2}
\end{array}\right]=\left[\begin{array}{l}
-3.77 \\
-4.81
\end{array}\right],
\end{aligned}
$$

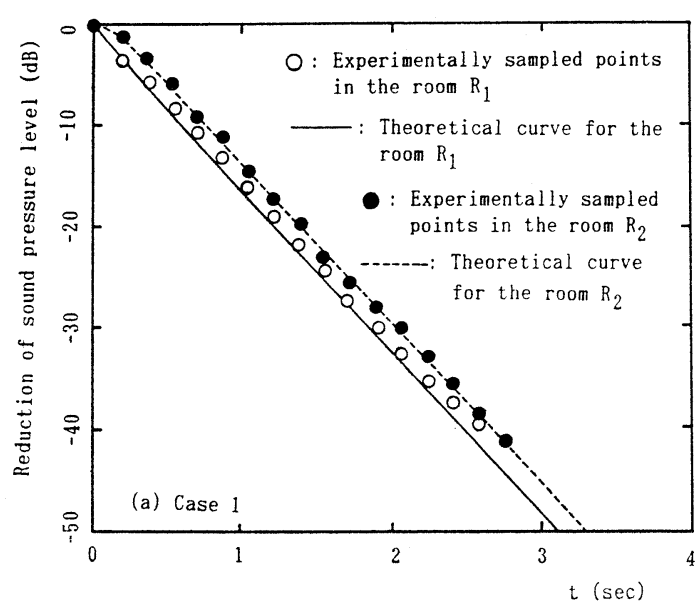

Fig. 4 Comparison between experimentally sampled points and theoretically estimated curves for decay curves of reverberation in the acoustically coupled room in Case 1.
(Case 3)

$$
\begin{aligned}
\boldsymbol{E}_{1} & =\left[\begin{array}{l}
2.40 \times 10^{-3} \\
9.12 \times 10^{-5}
\end{array}\right], \quad \boldsymbol{E}_{2}=\left[\begin{array}{l}
9.12 \times 10^{-5} \\
4.26 \times 10^{-3}
\end{array}\right], \\
A & =\left[\begin{array}{cc}
-3.87 & 0.083 \\
0.169 & -4.44
\end{array}\right], \quad\left[\begin{array}{l}
\lambda_{1} \\
\lambda_{2}
\end{array}\right]=\left[\begin{array}{l}
-3.84 \\
-4.46
\end{array}\right] .
\end{aligned}
$$

In order to confirm the effectiveness of the proposed method, the comparisons are given for the above cases in Figs. 4, 5 and 6 between experimentally sampled points and theoretically estimated curves.

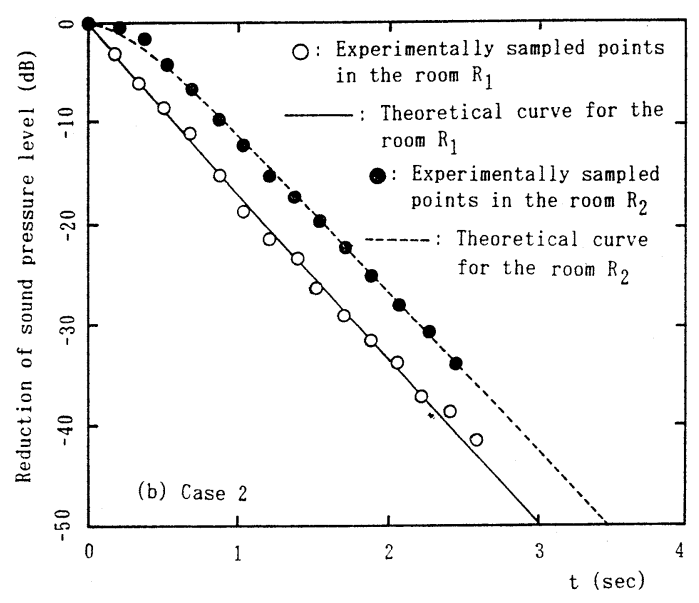

Fig. 5 Comparison between experimentally sampled points and theoretically estimated curves for decay curves of reverberation in the acoustically coupled room in Case 2.

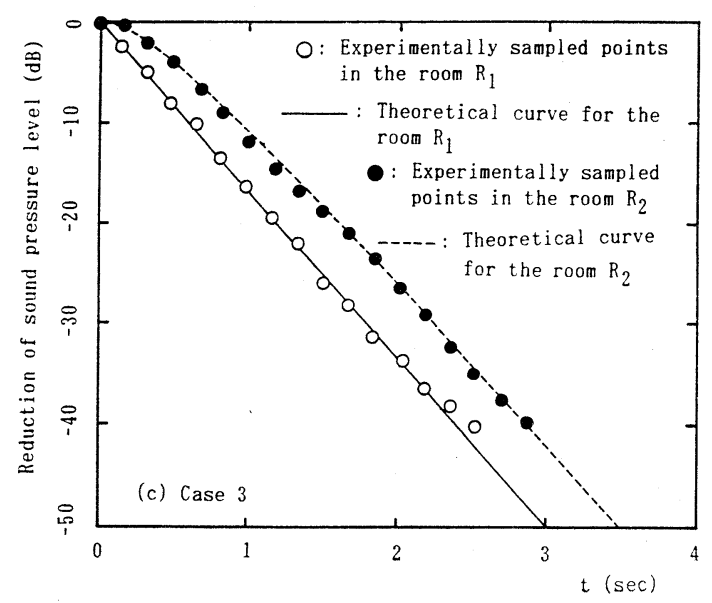

Fig. 6 Comparison between experimentally sampled points and theoretically estimated curves for decay curves of reverberation in the acoustically coupled room in Case 3. 


\section{OHTA et al.: SYSTEM-THEORETICAL EVALUATION METHOD OF COUPLED ROOM}

All of theoretically estimated curves fairly agree with the corresponding experimentally sampled points. Furthermore, it is found that the looser acoustical power flow interaction between two rooms $R_{1}$ and $R_{2}$ becomes, the larger the differences between the curves of $R_{1}$ and $R_{2}$ become.

\subsection{Evaluation Result on Reverberation Time}

The evaluation result of reverberation time by using the above six different methods are shown in Tables 1, 2 and 3 for the above three cases. Here, $T_{1}$ and $T_{1}$ denote two reverberation times of room $R_{1}$ and room $R_{2}$ respectively. That is, we get experimentally two observed values $T_{1}=4.1 \mathrm{~s}$ and $T_{2}=2.8 \mathrm{~s}$ by evaluating the slope of respective decay curve when the rooms are absolutely isolated from each other. Blanks in these tables denote that reverberation times are not computable.

The following characteristic points can be found from the above results:

(1) In the room having the speaker, the results by six different methods make to remarkable difference. But in the other room, the results show a relatively great difference.

(2) Results based on two Methods 2 and 6 agree with each other in all cases.

Table 1 Reverberation times in Care 1.

\begin{tabular}{lccccc}
\hline & \multicolumn{2}{c}{ Speaker in $\mathrm{R}_{1}$} & & \multicolumn{2}{c}{ Speaker in $\mathrm{R}_{2}$} \\
\cline { 2 - 3 } \cline { 5 - 6 } & $T_{1}$ & $T_{2}$ & & $T_{1}$ & $T_{2}$ \\
\hline Method 1 & 3.83 & 3.83 & & 3.69 & 3.69 \\
Method 2 & 3.78 & 3.93 & & 3.93 & 3.62 \\
Method 3 & 3.79 & 3.79 & & 3.79 & 3.79 \\
Method 4 & 3.04 & & & 2.21 \\
Method 5 & 3.44 & 5.25 & & 3.93 & 3.62 \\
Method 6 & 3.78 & 3.93 & & 3.93 & 3.62 \\
\hline
\end{tabular}

Table 2 Reverberation times in Case 2.

(s)

\begin{tabular}{lcclcc}
\hline & \multicolumn{2}{c}{ Speaker in $\mathrm{R}_{1}$} & & \multicolumn{2}{c}{ Speaker in $\mathrm{R}_{2}$} \\
\cline { 2 - 3 } \cline { 5 - 6 } & $T_{1}$ & $T_{2}$ & & $T_{1}$ & $T_{2}$ \\
\hline Method 1 & 3.68 & 3.68 & & 3.46 & 3.46 \\
Method 2 & 3.63 & 4.07 & & 4.07 & 3.34 \\
Method 3 & 3.67 & 3.67 & & 3.67 & 3.67 \\
Method 4 & 3.49 & & & & 3.04 \\
Method 5 & 3.53 & 6.54 & & 6.54 & 3.08 \\
Method 6 & 3.63 & 4.07 & & 4.07 & 3.34 \\
\hline
\end{tabular}

Table 3 Reverberation times in Case 3.

(s)

\begin{tabular}{cccccc}
\hline & \multicolumn{2}{c}{ Speaker in $\mathrm{R}_{1}$} & & \multicolumn{2}{c}{ Speaker in $\mathrm{R}_{2}$} \\
\cline { 2 - 3 } \cline { 5 - 6 } & $T_{1}$ & $T_{2}$ & & $T_{1}$ & $T_{2}$ \\
\hline Method 1 & 3.59 & 3.59 & & 3.21 & 3.21 \\
Method 2 & 3.59 & 4.11 & & 4.11 & 3.15 \\
Method 3 & 3.59 & 3.59 & & 3.59 & 3.59 \\
Method 4 & 3.57 & & & & 3.12 \\
Method 5 & 3.58 & 6.70 & & 6.70 & 3.12 \\
Method 6 & 3.59 & 4.11 & & 4.11 & 3.15 \\
\hline
\end{tabular}

(3) In the room having the speaker, the resultant values from all methods in Case 3 are smallest.

(4) In the room having the speaker, the resultant values from all methods for $R_{1}$ in Case 1 are closest to $4.1 \mathrm{~s}$ of single room with no mutual power flow interaction. On the other hand, the resultant values from all methods for $\mathbf{R}_{2}$ in Case 3 are closest to $2.8 \mathrm{~s}$ of a single room $R_{2}$ with no mutual power flow interaction.

\section{CONCLUSION}

In this paper, the evaluation methods of reverberation time for a coupled room system have been newly proposed on trial from various type systemtheoretical viewpoints. More concretely, first after introducing the system equation for this coupled room system, six different evaluation methods of reverberation times for this coupled room system have been proposed under the introduction of various type evaluation criteria.

Finally, the measurement of reverberation times in the actual acoustic system with two reverberant rooms coupled acoustically each other has been carried out to clarify the effectiveness of the proposed evaluation methods.

Since such system-theoretical trials in this paper for evaluating the reverberation time of a coupled room system are at early stage of study especially in the field of room acoustics, the chief purpose of this paper has been focused on principally finding how to evaluate a coupled room system-theoretically.

There still remain our future researches for applying these evaluation methods to many of the actual coupled rooms and determining which method is most effective to explain the actual reverberation characteristics of a coupled room in close connection with variety of the actual engineering needs. 


\section{ACKNOWLEDGMENTS}

The authors wish to express their special thanks to Mr. Yoshihisa Hamada of Shikoku Electric Company Ltd. and Prof. K. Hatakeyama of Kinki University for their helpful assistance and advice.

\section{REFERENCES}

1) P. M. Morse and K. U. Ingard, Theoretical Acoustics (McGraw-Hill, New York, 1968), Chap. 10.

2) H. Kuttruff, Room Acoustics, 2nd ed. (Applied Science Publishers Ltd., London, 1979), Chap. 3.

3) C. Thompson, "On the acoustics of a coupled space," J. Acoust. Soc. Am. 75, 707-714 (1984).

4) A. Craggs and G. Stead, "Sound transmission between enclosures-A study using plate and acoustic finite elements," Acustica 35, 89-98 (1976).

5) K. Fujiwara and Y. Makita, "On the sound propagation through the stair case," J. Acoust. Soc. Jpn. (J) 32, 615-617 (1976) (in Japanese).

6) H. Date, "Reverberation time of a room partitioned by curtains," J. Acoust. Soc. Jpn. (J) 16, 83-86 (1960) (in Japanese).

7) A. Trochidis, "Reverberation time of Byzantine churches of Thessaloniki," Acustica 51, 299-301 (1982).

8) J. E. Brooks and G. Maidanik, "Loss and coupling loss factors of two coupled dynamic systems," J. Sound Vib. 55, 315-323 (1977).

9) M. J. Crocker and A. J. Price, "Sound transmission using statistical energy analysis," J. Sound Vib. 9, 469-486 (1969).

10) M. Ohta, K. Hatakeyama, and H. Yamada, "A trial of probabilistic evaluation for acoustically coupled room contaminated by an arbitrary random noiseResponse probability prediction and parameter identification by use of a wide-sense digital filter," J. Acoust. Soc. Jpn. (J) 48, 365-374 (1992) (in Japanese).

11) M. Ohta and H. Iwashige, "A new establishment of evaluation theory on the reverberation characteristics for a coupled room and an experiment," Proc. Autumn Meet. Acoust. Soc. Jpn. 1-4-6, 467-468 (1984) (in Japanese).

12) M. Ohta and H. Iwashige, "A new systematic evaluation on the reverberation characteristics for a general coupled room and an experiment," Proc. Autumn Meet. Acoust. Soc. Jpn. 3-4-10, 655-656 (1987) (in Japanese).

13) H. Yamada, M. Ohta, and H. Iwashige, "A systemtheoretical evaluation method for the reverberation time of acoustically coupled room," Proc. InterNoise 92, 703-706 (1992).

14) M. Ohta, H. Yamada, and H. Iwashige, "System theoretical evaluation method for the reverberation characteristics of a coupled room-Synthetic eval- uation methods by matrix measure and individual evaluation methods-," Proc. Spring Meet. Acoust. Soc. Jpn. 1-4-4, 677-678 (1992) (in Japanese).

15) Nippon-Onkyozairyo-Kyokai, Ed., Handbook of Noise Control (Gihodo, Tokyo, 1982), p. 624 (in Japanese).

16) M. Ohta, "Various dynamical methods of state estimation in the environmental noise and vibration systems-I-I-Methodological attitude and establishment of general theory-,", Syst. Control 23, 571577 (1979) (in Japanese).

17) M. Ohta, "Various dynamical methods of state estimation in the environmental noise and vibration systems-II-State estimation theory of energy system and its application to dwelling environmental noise-,"' Syst. Control 23, 702-709 (1979) (in Japanese).

18) H. Iwashige and M. Ohta, "A study on sound transmission loss of double-walls having several types of geometrical section by use of the improved statistical energy analysis method," J. Acoust. Soc. Jpn. (J) 36, 447-458 (1980) (in Japanese).

19) H. Iwashige and M. Ohta, "A practical method of estimating sound transmission loss of double walls -A proposal and its application to the popular case of light panels and air gap," Acustica 48, 79101 (1981).

20) H. Iwashige and M. Ohta, "A study on sound transmission loss of general N-fold walls by use of the improved statistical energy analysis method," J. Acoust. Soc. Jpn. (J) 37, 484-493 (1981) (in Japanese).

\section{APPENDIX: EXPERIMENTAL VERIFICATION OF COMPLETE DIFFUSENESS OF ROOMS}

The complete diffuseness of rooms $R_{1}$ and $R_{2}$ can be experimentally verified as follows.

Let us employ Eq. (4a) as a kind of indices for evaluating the complete diffuseness of rooms $R_{1}$ and $R_{2}$. In Case 2, the theoretically calculated values of $a_{12}$ and $a_{21}$ by using Eq. (4a) are 0.307 and 0.627 , respectively. On the other hand, the experimentally estimated values $\hat{a}_{12}$ and $\hat{a}_{21}$ from the actually observed data are 0.303 and 0.618 , respectively, as mentioned before. These values are very close each other. In Case 3, the estimated values of the transmission loss of the lauan board of $3 \mathrm{~mm}$ thickness are $14 \mathrm{~dB}$ from Eq. (4a) by using both of estimated values $\hat{a}_{12}=0.083$ and $\hat{a}_{21}=0.169$. On the other hand, the experimental transmission loss of the lauan board of $3 \mathrm{~mm}$ thickness from the reference 15 is the same $14 \mathrm{~dB}$ for a center frequency $500 \mathrm{~Hz}$. These results suggest that two rooms $R_{1}$ and $R_{2}$ can be respectively regarded as completely diffuse sound 


\section{OHTA et al.: SYSTEM-THEORETICAL EVALUATION METHOD OF COUPLED ROOM}

fields.

Moreover, it is noteworthy that the same rooms had been already employed and then verified very often their complete diffuseness in our other previous researches. ${ }^{16-20)}$

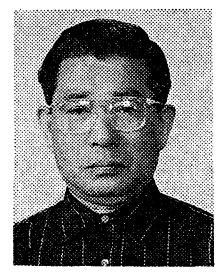

Mitsuo Ohta was born in 1928. He received the B.S. degree on physics and the Ph.D. degree in physics from the Kyoto University in 1952 and 1961, respectively. After working as a Research Assistant and an Associate Professor in Kobe and Fukui Universities, and as a Professor in Fukui and Hiroshima Universities, he has been a Professor at the Faculty of Engineering, Kinki University, since 1990. His research fields are the studies of microwave, dielectric measurement, ultra-sound, electro-magnetic-wave propagation, information processing, control theory, probabilistic engineering, sound-and-vibration control and digital filter. He got the prize in praise of his paper from the Acoustical Society of Japan and the authorized license of technology from the Institute of Noise Control Engineers of Japan. He is a member of the Acoustical Society of Japan, the Society of Instrument and Control Engineers, the Institute of Systems, Control and Information Engineers, the Institute of Electronics, Information and Communication Engineers and the Institute of Electrical Engineers of Japan.

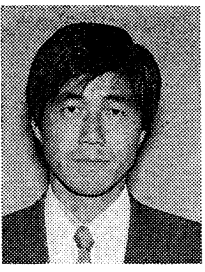

Hiroshi Yamada was born in 1958. He received the B.E., M.E. and Dr. engineering degrees from Hiroshima University in 1980, 1982, and 1985, respectively. After working as a Lecturer and an Associate Professor in Oshima National College of Maritime Technology, Yamaguchi, Japan, he has been an Associate Professor at the Faculty of Engineering, University of East Asia, Shimonoseki, Japan, since 1991. His research interests include prediction and evaluation of environmental noise, signal processing, stochastic system theory and statistical automatic control engineering. $\mathrm{He}$ is a member of the Acoustical Society of Japan, the Institute of Noise Control Engineers, the Society of Instrument and Control Engineers, the Institute of Systems, Control and Information Engineers and the Institute of Electronics, Information and Communication Engineers.

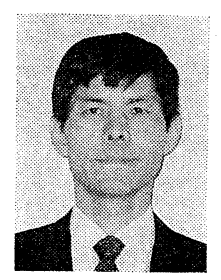

Hirofumi Iwashige was born in Iwakuni, Japan, on May 26, 1944. He received the B.A., M.A. degrees in Architecture in 1967, 1969 respectively, and the Ph.D. degree in 1982 from Hiroshima University, Higashi-Hiroshima, Japan. Since April 1969 he worked at Hiroshima University, where he is a Professor of Housing. From June 1969 to June 1971, he studied at University of Aix-Marseille, France, and from October 1993 to August 1994, he was a visiting scholar at Cornell University, Ithaca, NY. His research interest includes architectural acoustics and environmental factors. Dr. Iwashige is a member of the Acoustical Society of Japan and Architectural Institute of Japan. 